\title{
Construction der Fläche zweiten Grades durch neun Punkte.
}

(Nach den hinterlassenen Manuscripten Jacob Steiners dargestellt von Herrn C. F. Geiser in Zürich.)

Die Aufgabe eine Fläche zweiten Grades durch neun im Raume beliebig gegebene Punkte zu legen, ist bekanntlich durch die Herren Hesse (Bd.24 dieses Journals), Seydewitz (Bd.9 des Grunertschen Archivs) und Schröter (Bd. 62 dieses Journals) gelöst worden. In den hinterlassenen Manuscripten Steiners ist nun ein mit kurzen Notizen versehenes Quartblatt vorhanden, welches zeigt, dass Steiner bereits im Jahre 1836 zwei verschiedene Constructionen dieser Fläche gefunden hatte, die er aber nicht veröffentlichte, weil die zugehörigen Beweise nicht vollständig und einfach genug und die Constructionen nicht linear waren. Während, wie es scheint, die von Steiner als zweite dieser Lösungen bezeichnete Construction nicht auf die nöthige Einfachheit gebracht werden kann, und sich desshalb zur Veröffentlichung nicht eignet, ist es gelungen, mit einigen Abänderungen und Vervollständigungen die erste derselben in eine Form zu bringen, welche, trotzdem die gesuchte Fläche nicht linear hergestellt wird, doch mit so geringen Mitteln zum Ziele führt, als man üherhaupt bei der complicirten Aufgabe erwarten darf. Ihrer Darstellung ist die nachfolgende kurze Nittheilung gewidmet.

Wenn den neun gegebenen Punkten in einer beliebigen Reihenfolge die Zahlen (1) bis (9) zugefügt werden, so lege man zuerst die Ebenen (123), (456), (789), die man resp. mit I, II, III bezeichne; ihr gemeinschaftlicher Durchschnittspunkt heisse $S$. Die Schnittgeraden von II und III, III und I, I und II, welche $A, B, C$ heissen sollen, stehen nun zu der gesuchten Fläche $f_{2}$ in der nachstehenden Beziehung: Jede der Ebenen I, II, III hat mit $f_{2}$ einen Kegelschnitt gemein, und für diese Kegelschnitte zu je zweien genommen, sind die Geraden $A, B, C$ gemeinschaftliche (reelle oder ideelle) Sehnen; kann man umgekehrt durch die Punkte 123, 456, 789 drei Kegelschnitte legen, für welche $A, B, C$ gemeinschaftliche Sehnen sind, so liegen diese drei Kegelschnitte auf $f_{2}$.

Man betrachte zunächst nur die Punkte (1) bis (8). Die Gerade (23) trifft $B$ und $C$ resp. in Punkten $b$ und $c$, von denen $c$ mit $(4)$, (5) und (6) 
eine Kegelschnittschaar bestimmt. Ein willkürlicher Kegelschnitt der Schaar schneidet auf $C$ ausser $c$ einen Punkt $c^{\prime}$ aus, ferner ergiebt dieser Kegelschnitt (456c $c^{\prime}$ ) auf $A$ zwei Punkte $a$ und $a^{\prime}$, welche mit (7), (8) und $b$ einen neuen Kegelschnitt in der Ebene III bestimmen, der die Gerade $B$ ausser in $b$ noch in einem Punkte $b^{\prime}$ schneidet. Der Punkt $b^{\prime}$ ist durch den Punkt $c^{\prime}$ bestimmt; wenn $c^{\prime}$ auf der Geraden $C$ sich bewegt, so durchläuft $b^{\prime}$ die Gerade $B$, und da zu jedem $c^{\prime}$ stets ein, aber nur ein $b^{\prime}$ gehört, und umgekehrt, so sind $B$ und $C$ hinsichtlich der Punkte $b^{\prime}$ und $c^{\prime}$ projectivisch. Aber $b^{\prime}$ und $c^{\prime}$ gehen gleichzeitig durch $S$, d. h. $B$ und $C$ sind zugleich perspectivisch, und alle Verbindungsgeraden entsprechender $b^{\prime}$ und $c^{\prime}$ laufen durch einen und denselben in der Ebene I gelegenen Punkt $M$. Fassen wir jetzt die Geraden (23) und (M1) als Kegelschnitt $K_{1}$ auf, der ganz in der Ebene I liegt, und welcher einen bestimmten Punkt $c^{\prime}$ auf $C$ ergiebt, so erhält man in der angegebenen Weise zu diesem einen Kegelschnitt $K_{2}$ in der Ebene II und einen Kegelschnitt $K_{3}$ in der Ebene III. Diese drei Kegelschnitte haben die Geraden $A, B, C$ zu gemeinschaftlichen Sehnen, und gehören demzufolge einer Fläche zweiten Grades $F_{2}$ an, welche durch die Punkte (1) bis (8) geht.

Wiederholt man dieses ganze Verfahren, indem man stalt von der Geraden (23) nun von der Geraden (31) ausgeht, so erhält man in den Ebenen I, II, III drei neue Kegelschnitte $K_{1}^{\prime}, K_{2}^{\prime}, K_{3}^{\prime}$, die wieder auf einer Fläche $F_{2}^{\prime}$ liegen, welche die Punkte $(1)$ bis $(8)$ enthält. Die Flächen $F_{2}$ und $F_{2}^{\prime}$ schneiden sich in einer durch die Punkte (1) bis (8) gehende Raumcurve, durch welche unendlich viele Flächen zweiten Grades gehen, unter denen sich auch $f_{2}$ befindet, welche die Punkte (1) bis (9) enthält. Diese Schnittcurve hat mit jeder der Ebenen I, II, III vier Punkte gemein, welche resp. die Durchschnitte von $K_{1}$ und $K_{1}^{\prime}, K_{2}$ und $K_{2}^{\prime}, K_{3}$ und $K_{3}^{\prime}$ sind. Schneiden sich nun $K_{3}$ und $K_{3}^{\prime}$ ausser in (7) und (8) noch in $\gamma$ und $\gamma^{\prime}$, so gehört der Kegelschnitt $\left(78 \gamma \gamma^{\prime} 9\right)$ der gesuchten Fläche $f_{2}$ an, von welcher natürlich sofort noch zwei andere Kegelschnitte zu finden sind. Damit darf die gestellte Aufgabe als gelöst betrachtet werden, und es ist nur noch hinzuzufügen, dass diese Lösung zugleich die Construction derjenigen Raumcurve vierten Grades ergiebt, welche der Durchschnitt zweier Flächen zweiten Grades ist, und durch acht gegebene Punkte im Raume geht.

Zürich, im Januar 1867. 